\title{
PAAm-Kappa Carrageenan Composites: Drying and Swelling with Various Kappa Carrageenan Contents
}

\author{
G. AKIn EvingüR ${ }^{a}$ AND Ö. PEKCAN ${ }^{b}$ \\ ${ }^{a}$ Piri Reis University, 34940 Tuzla, İstanbul, Turkey \\ ${ }^{b}$ Kadir Has University, 34083, Cibali, İstanbul, Turkey
}

\begin{abstract}
The purpose of this study is to discuss the role of kappa-carrageenan $(\kappa$-car) in the drying and swelling of polyacrylamide (PAAm) $-\kappa$-car composite. The fluorescence intensity of pyranine increased and decreased as drying and swelling time were increased respectively for all samples. The desorption coefficient, $D_{\mathrm{d}}$, decreased as $\kappa$-car contents were increased for a given temperature during drying. However, cooperative diffusion coefficient, $D_{\mathrm{s}}$, increased as $\kappa$-car contents were increased during swelling at a given temperature.
\end{abstract}

PACS: 82.35.Lr, 83.80.Kn, 87.15.Vv, 87.15.Zg, 87.64.kv

\section{Introduction}

Chemical gels doped with biological gels are a new class of composite materials. Low and high water sorption properties of composites are important for the use of sorbents in many applications of biomaterials and separation operations in biotechnology, drug delivery systems, processing of agricultural products, sensors and actuators [1]. Absorbent and adhesive properties of $\kappa$ -carrageenan graft PAAm copolymer hydrogels were examined by using IR spectroscopy, optical microscopy, thermogravimetrical analysis and rheometry. A microwave induced one-pot route for graft copolymerization of AAm onto carrageenan initiated by potassium per sulfate (KPS) was used. The resultant copolymer hydrogel exhibited adhesive and super absorbent properties [1]. Super absorbent hydrogels of kappa-carrageenan-graft-polyacrylamide were also synthesized by a simultaneous irradiation technique using $\gamma$-rays as the energy source, initiator and crosslinker under various conditions. The swelling behavior of the super absorbent polymers was related to their chemical structure, chemical composition, the absorbing environment and the nature of the solution [2].

In this study, the steady state fluorescence technique was introduced for studying the drying and swelling of disc shaped PAAm- $\kappa$-car composite gels with various $\kappa$-car contents. Composite gels were prepared at $80^{\circ} \mathrm{C}$ with pyranine $(\mathrm{Py})$ doped as a fluorescence probe. Scattered light, $I_{\mathrm{sc}}$, and fluorescence intensities, $I$ were monitored during drying of these gels. The fluorescence intensity of pyranine increased and decreased as drying and swelling time were increased for all samples, respectively. The Stern-Volmer equation combined with moving boundary and the Li-Tanaka models were used to explain the behavior of $I$ during drying and swelling, re- spectively. It is found that desorption coefficient, $D_{\mathrm{d}}$, decreased as $\kappa$-car contents were increased for a given temperature during drying. However cooperative diffusion coefficient, $D_{\mathrm{s}}$, increased as $\kappa$-car contents were increased during swelling at a given temperature.

\section{Theoretical consideration}

\subsection{Stern-Volmer kinetics}

This model is based on the variations of quantum yields of photophysical processes such as fluorescence, phosphorescence and photochemical reactions with the concentration of a given quencher.

According to the Stern-Volmer law, fluorescence intensity can be written as [3]:

$$
\frac{I_{0}}{I}=1+k_{q} \tau_{0}[Q] .
$$

Here, $k_{q}$ is quenching rate constant, $\tau_{0}$ is the lifetime of the fluorescence probe with no quenching taking place, and $[Q]$ is the quencher concentration and $I_{0}$ is the fluorescence intensity for zero quencher content. This relation is called the Stern-Volmer equation. Equation (2.1) becomes

$$
I \approx I_{0}\left(1-\tau_{0} k_{q}[Q]\right) .
$$

If one integrates Eq. (2.2) over the differential volume $(\mathrm{d} v)$ of the sample from the initial, $a_{0}$, to final, $a_{\infty}$, thickness, then reorganization of the relation produces the following useful equation:

$$
W=\left(1-\frac{I}{I_{0}}\right) \frac{v}{k_{q} \tau_{0}} .
$$

Here, the amount of water desorption, $W$, is calculated over differential volume by replacing $Q$ with $W$. 


\subsection{Moving boundary model}

Diffusion in a system with a moving boundary occurs in two distinct regions separated by a moving interface. When the diffusion coefficient is discontinuous at a concentration $c$, i.e. the diffusion coefficient is zero below $c$ and constant and finite above $c$, then the total amount, $M_{\mathrm{t}}$, of diffusing substance desorbed from unit area of a plane sheet of thickness $a$ at time $t ; D_{\mathrm{d}}$ is a diffusion coefficient at concentration $c_{1}$. Here $M_{\mathrm{f}}=a c_{1}$ is the equilibrium value of $M_{\mathrm{t}}$ [4]. If one assumes that the diffusion coefficient of polymer segments in the gel is negligible compared to the desorption coefficient, $D_{\mathrm{d}}$, of water, then equation can be written as follows:

$$
\frac{W}{W_{\mathrm{f}}}=2\left(\frac{D_{\mathrm{d}}}{\pi a^{2}}\right)^{1 / 2} t^{1 / 2} .
$$

Here it is assumed that $M_{\mathrm{t}}$ is proportional to the amount of water molecules released, $W$, at time $t$.

\subsection{Li-Tanaka model}

The kinetics of the swelling of a gel is completely described by the behavior of the displacement vector as a function of space and time. Li and Tanaka showed that the equation of motion is given by

$$
f \frac{\partial \boldsymbol{u}}{\partial t}=\nabla \cdot \boldsymbol{\sigma}
$$

where $\boldsymbol{u}$ is the displacement vector measured from the final equilibrium location after the gel is fully swollen $(\boldsymbol{u}=0$ at $t=\infty)$. Equation (2.5) is solved with cylindrical coordinates. In the limit of large $t$, or if $\tau_{1}$ is much larger than the rest of $\tau_{n}$, all higher terms $(n \geq 2)$ in Eq. (2.6) can be omitted and the swelling kinetics is given by the following relation:

$$
\frac{W}{W_{\mathrm{f}}}=1-B_{1} \exp \left(-t / \tau_{1}\right)
$$

where $\tau_{1}$ is related to the cooperative diffusion coefficient, $D_{\mathrm{s}}$, at the surface of a gel disc by

$$
D_{\mathrm{s}}=\frac{3 a_{\mathrm{f}}^{2}}{\tau_{1 I} \alpha_{I}^{2}},
$$

where $\alpha_{I}$ is a function of $R$ only [5], and $a_{\mathrm{f}}$ stands for the half thickness of the gel in the final equilibrium state. Hence, $D_{\mathrm{s}}$ can be calculated.

\section{Experimental consideration}

Composite gels were formed by free radical copolymerization as follows: $0.71 \mathrm{~g}$ of acrylamide (AAm, Merck), $0.01 \mathrm{~g}$ of $N, N^{\prime}$-methylenebisacrylamide (BIS, Merck), $0.008 \mathrm{~g}$ of ammonium persulfate (APS, Merck) and $2 \mu \mathrm{l}$ of tetramethylethylenediamine (TEMED, Merck) were dissolved in $5 \mathrm{ml}$ distilled water $(\mathrm{pH} 6.5)$ by heating. The heated mixture solution was held at $80^{\circ} \mathrm{C}$. Then varying amounts of carrageenan $(0.5,1,1.5,2,2.5$ and $3(\mathrm{w} / \mathrm{v}) \%)$ were added [6]. Py concentration was kept constant at $4 \times 10^{-4} \mathrm{M}$, for all experiments. The solution was stirred
(200 rpm) for 15 min to achieve a homogeneous solution. All samples were deoxygenated by bubbling nitrogen for 10 min just before polymerization process. The drying and swelling experiments of disc shape PAAm- $\boldsymbol{K}$-car composite gels prepared were performed in air and in water, respectively at temperatures of $30,40,50$ and $60^{\circ} \mathrm{C}$.

The fluorescence intensity measurements were carried out using a model LS-50 spectrometer from Perkin-Elmer, equipped with a temperature controller. All measurements were made at a $90^{\circ}$ position and spectral bandwidths were kept at $5 \mathrm{~nm}$. Disc-shaped gel samples were placed on the wall of a $1 \mathrm{~cm}$ path length square quartz cell filled with air and/or water for the drying and swelling experiments. Composite gels were excited at $340 \mathrm{~nm}$ during in situ experiments and emission intensities of the pyranine were monitored at $427 \mathrm{~nm}$ as a function of drying and swelling time. As the water released began to increase, the fluorescence intensity, $I_{\mathrm{em}}$, increased and the scattered light intensity, $I_{\mathrm{sc}}$, decreased. During swelling, however, $I_{\mathrm{em}}$ and $I_{\mathrm{sc}}$ behaved in exact reverse manner to the drying behavior.

\section{Results and discussion}

Figure 1a and $\mathrm{b}$ shows the emission spectra of pyranine from PAAm- $\kappa$-car composite gel during the drying and swelling process at $50{ }^{\circ} \mathrm{C}$ for $3(\mathrm{w} / \mathrm{v}) \% \kappa$-car content samples. It can be seen in Fig. 1a that as the water release is increased, fluorescence intensity, $I_{\mathrm{em}}$, increases and the scattered light intensity, $I_{\mathrm{sc}}$, decreases. However, Fig. $1 \mathrm{~b}$ shows the reverse behavior for $I_{\mathrm{em}}$ and $I_{\mathrm{sc}}$ during swelling of PAAm- $\boldsymbol{k}$-car composite gel. Since the decrease in $I_{\mathrm{sc}}$ corresponds to the decrease in turbidity of the drying gel, the corrected fluorescence intensity, $I$, is defined as $I_{\mathrm{em}} / I_{\mathrm{sc}}$ i.e. as the drying time, $t$, is increased, quenching of excited pyranines decreases due to increase of water release from the drying $\mathrm{PAAm}^{-} \boldsymbol{\kappa}$-car composite gel.

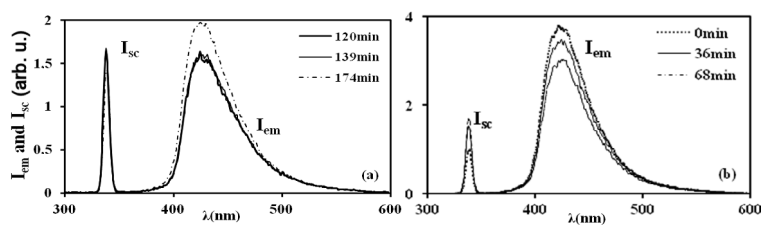

Fig. 1. Emission spectra of pyranine from the hydrogel prepared with $3(\mathrm{w} / \mathrm{v}) \% \kappa$-car content samples at $50^{\circ} \mathrm{C}$ during the (a) drying and (b) swelling process, and each curve indicates the drying and swelling times in different minutes, respectively.

In order to quantify these results the collisional type of quenching mechanism may be proposed for the fluorescence intensity, $I$, from the gel samples during the drying process by using Eq. (2.3). For the dry composite gel, $W$ can be calculated by using Eq. (2.3) and the measured $I$ values at each drying step. The plots of $W$ versus $t$ at various $\kappa$-car content samples are presented in Fig. 2a where the fit of the data to Eq. (2.4) produced the desorption 
coefficient, $D_{\mathrm{d}}$, as shown in Fig. $2 \mathrm{~b}$. It is obvious that less $\kappa$-car content gel dries faster than high $\kappa$-car content gel. It is seen that $D_{\mathrm{d}}$ values decreased as the $\boldsymbol{\kappa}$-car content was increased.

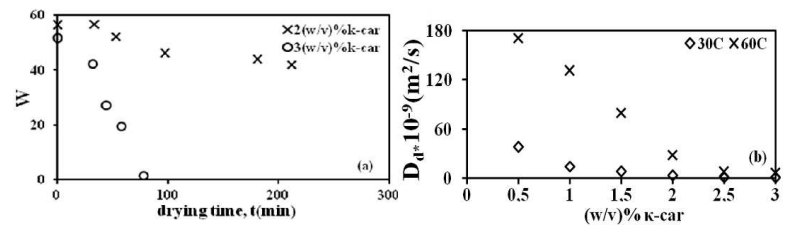

Fig. 2. The plots of (a) water release, $W$, versus drying time, $t$, for 2 and $3(\mathrm{w} / \mathrm{v}) \% \kappa$-car, and (b) desorption diffusion coefficients versus $k$-car content measured by fluorescence technique at 30 and $60^{\circ} \mathrm{C}$ temperatures, respectively.

For the swollen composite gel, $W$ can be calculated by using Eq. (2.3) and the measured $I$ values, at each swelling step [3]. Once $k_{q}$ values are measured, the water uptakes, $W$, can be calculated from the measured $\tau_{1 I}$ values at each swelling step. Here, it is assumed that the $k_{q}$ values do not vary during the swelling processes, i.e. the quenching process solely originates from the water molecules. Plots of water uptake, $W$, versus swelling time are presented in Fig. 3a. The logarithmic form of the data in Fig. 3a was fitted to the following relation produced from Eq. (2.6)

$$
\ln \left(1-\frac{W}{W_{\mathrm{f}}}\right)=\ln B_{1}-\frac{t}{\tau_{1 I}} .
$$

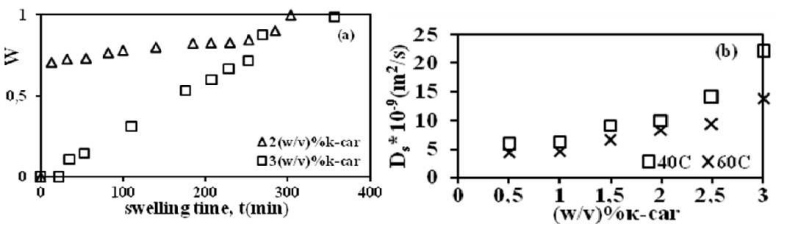

Fig. 3. The plots of (a) water uptake, $W$, versus swelling time, $t$, for 2 and $3(\mathrm{w} / \mathrm{v}) \% \kappa$-car, and (b) cooperative diffusion coefficients versus $\kappa$-car content measured by fluorescence technique at 40 and $60^{\circ} \mathrm{C}$ temperatures, respectively.

Here, $\tau_{1 I}$ is the time constant, measured by fluorescence technique and $B_{1}$ is related to the ratio of the shear modulus, $\mu$, and longitudinal osmotic modulus, $M$. Using Eq. (4.1) linear regression of the curves in Fig. 3a provided us with $B_{1}$ and $\tau_{1 I}$ values. Taking into account the dependence of $B_{1}$ on $R$, one obtains $R$ values and from the $\alpha_{1}-R$ dependence $\alpha_{1}$ value was produced. The experimental determination of these values were based on the method described by Li and Tanaka [5]. Then, using Eq. (2.7), cooperative diffusion coefficients $D_{\mathrm{s}}$ were determined for these disc-shaped gels and found to be around $10^{-9} \mathrm{~m}^{2} / \mathrm{s}$ as shown in Fig. $3 \mathrm{~b}$.

\section{Conclusion}

This work has presented that the fluorescence method can be used for monitor drying and swelling behaviors of PAAm- $\kappa$-car composite gel prepared with various $\kappa$-car content. It was observed that high $\kappa$-car content composite gels dry much slower as the result of having larger $D_{\mathrm{d}}$ coefficients for all measurements compared to low $\kappa$-car content composite gels. The results were interpreted in terms of the swelling time constants; $\tau_{1 I}$ decreased and $D_{\mathrm{s}}$ increased as the $\boldsymbol{\kappa}$-car contents increased. It was observed that high $\kappa$-car content composite gels swell much faster as the result of having larger of $D_{\mathrm{s}}$ coefficients for all measurements in comparison to low $\kappa$-car content composites for a given temperature.

\section{Acknowledgments}

Experiments were done in Spectroscopy Lab. in the department of Physics Engineering of İstanbul Technical University

\section{References}

[1] R. Meena, K. Prasad, G. Mehta, A.K. Siddhanta, J. Appl. Polym. Sci. 102, 5144 (2006).

[2] G.R. Bardajee, A. Pourjavadi, N. Sheikh, M.S. Amini-Fazl, Radiat. Phys. Chem. 77, 131 (2008).

[3] J.B. Birks, Photophysics of Aromatic Molecules, Wiley, Interscience, New York 1971.

[4] J. Crank, The Mathematics of Diffusion, Clarendon Press, Oxford 1975.

[5] Y. Li, T. Tanaka, J. Chem. Phys. 92, 1365 (1990).

[6] D. Kaya Aktaş, G.A. Evingur, Ö. Pekcan, J. Biomol. Struct. Dynam. 24, 83 (2006). 